\title{
CSR 2018 Special Issue on TOCS
}

\author{
Fedor V. Fomin ${ }^{1} \cdot$ Vladimir V. Podolskii $^{2,3}$
}

Published online: 16 November 2019

(C) Springer Science+Business Media, LLC, part of Springer Nature 2019

This special issue of Theory of Computing Systems consists of extended journal papers originally presented at the 13th International Computer Science Symposium in Russia (CSR 2018) held on June 6-10, 2018 in Moscow, Russia. The event was hosted by National Research University Higher School of Economics and chaired by Vladimir V. Podolskii. Preliminary versions of these papers presented at the conference appeared in LNCS 10846. The Program Committee, chaired by Fedor V. Fomin, invited several authors to submit extended journal versions of their papers to this special issue. All submissions were reviewed in accordance with customary high standards.

The CSR conference series is devoted to a broad scope of computer science topics.

A short description of the contributions in this issue follows.

The paper Recognizing Read-Once Functions from Depth-Three Formulas by Alexander Kozachinskiy (this paper received the Best Student Paper award at the conference) studies the complexity of deciding whether a given monotone Boolean function is read-once based on its low-depth representation. In particular, the paper shows that this problem is coNP-complete when the input formula is depth-3 read-2.

The paper The Clever Shopper Problem by Laurent Bulteau, Danny Hermelin, Dušan Knop, Anthony Labarre and Stéphane Vialette investigates a variant of the socalled Internet Shopping problem, where a customer wants to buy a list of products at the lowest possible total cost from shops which offer discounts when purchases exceed a certain threshold. In particular, the paper gives polynomial and FPT algorithms for some interesting special cases of the problem.

The paper Slopes of multidimensional subshifts by Emmanuel Jeandel, Etienne Moutot and Pascal Vanier concerns the directions of periodicity of multidimensional subshifts of finite type (SFTs) and of multidimensional effectively closed and sofic subshifts. The paper gives characterization of the sets of slopes of 2D and 3D subshifts.

Vladimir V. Podolskii

podolskii@mi-ras.ru

Fedor V. Fomin

fomin@ii.uib.no

1 Department of Informatics, University of Bergen, Bergen, Norway

2 Steklov Mathematical Institute, Moscow, Russia

3 National Research University Higher School of Economics, Moscow, Russia 
The paper Fixed-Parameter Tractable Algorithm and Polynomial Kernel for MaxCut Above Spanning Tree by Jayakrishnan Madathil, Saket Saurabh and Meirav Zehavi (this paper received the Best Paper award at the conference) studies Max-Cut Above Spanning Tree problem. The paper shows that this problem is fixed parameter tractable with respect to the size of the cut above the spanning tree bound.

The paper Quadratically Tight Relations for Randomized Query Complexity by Rahul Jain, Hartmut Klauck, Srijita Kundu, Troy Lee, Miklos Santha, Swagato Sanyal and Jevgēnijs Vihrovs investigates the problem of quadratically tightly approximating the randomized query complexity of Boolean functions. The paper introduces expectational certificate complexity, which is a quadratically tight bound on the zero-error randomized query complexity, and investigates whether the corruption bound quadratically approximates randomized query complexity.

The paper Complexity and Inapproximability Results for Parallel Task Scheduling and Strip Packing by Klaus Jansen, Sören Henning, Malin Rau and Lars Schmarje studies Parallel Task Scheduling with a constant number of machines. The paper shows that this problem is strongly NP-complete for the case of 4 machines. The paper also improves the lower bound for approximating pseudopolynomial Strip Packing.

The paper Grammar-based Compression of Unranked Trees by Adria Gascon, Markus Lohrey, Sebastian Maneth, Carl Philipp Reh, Kurt Sieber introduces forest straight-line as a compressed representation of unranked ordered node-labelled trees. The paper compares the succinctness of this representation with two other compression schemes for unranked trees: top dags and tree straight-line programs of firstchild/next sibling encodings. The paper shows that equality of unranked trees in the setting where certain symbols are associative and/or commutative can be tested in polynomial time.

The paper Periodicity in Data Streams with Wildcards by Funda Ergun, Elena Grigorescu, Erfan Sadeqi Azer, Samson Zhou investigates the problem of detecting periodic trends within a string, arriving in the streaming model, containing small number of wildcard characters. The paper gives upper and lower bounds on the space of one-pass and two-pass streaming algorithms for this problem.

Bergen and Moscow, September 2019 F.V. Fomin and V.V. Podolskii. 